\title{
Philosophiques
}

\section{Les objets sociaux}

\section{Barry Smith}

Volume 26, numéro 2, automne 1999

La critique de la raison en Europe centrale

URI : https://id.erudit.org/iderudit/004987ar

DOI : https://doi.org/10.7202/004987ar

Aller au sommaire du numéro

\section{Éditeur(s)}

Société de philosophie du Québec

\section{ISSN}

0316-2923 (imprimé)

1492-1391 (numérique)

Découvrir la revue

\section{Citer cet article}

Smith, B. (1999). Les objets sociaux. Philosophiques, 26(2), 315-347.

https://doi.org/10.7202/004987ar

\section{Résumé de l'article}

L'une des raisons qui expliquent l'intérêt renouvelé pour la philosophie autrichienne, et spécialement pour l'oeuvre de Brentano et de ses héritiers, est le fait que les philosophes analytiques ont eu un regain d'intérêt pour les problèmes traditionnels de la métaphysique. Il revient à Brentano, Husserl, et aux philosophes et psychologues qu'ils ont influencés, d'avoir attiré l'attention sur l'épineux problème de l'intentionnalité, qui consiste à rendre compte de la relation entre les actions et les objets ou, plus généralement, de la relation entre les environnements psychologiques des sujets cognitifs et les différents types d'environnements externes (physique, géographique, social) qu'ils habitent. Le présent article s'intéresse à cette version environnementale du problème de l'intentionnalité. Nous nous pencherons non seulement sur les travaux de Husserl et de Scheler, mais aussi sur ceux de Kurt Koffka et de Kurt Lewin en psychologie de la Gestalt. Nous tiendrons compte de l'influente théorie idéaliste subjective des environnements animaux mise de l'avant par J. von Uexküll, que nous comparerons avec une théorie réaliste de l'interaction organisme-environnement, théorie basée sur les écrits des psychologues écologistes J. J. Gibson et Roger Barker. Cette théorie réaliste sera alors exploitée en tant que fondement d'une ontologie des objets sociaux de différents types.
Ce document est protégé par la loi sur le droit d'auteur. L'utilisation des services d’Érudit (y compris la reproduction) est assujettie à sa politique d'utilisation que vous pouvez consulter en ligne.

https://apropos.erudit.org/fr/usagers/politique-dutilisation/ 


\title{
Les objets sociaux'
}

\author{
BARRY SMITH \\ University of Buffalo \\ Phismith@acsu.bufalo.edu
}

\begin{abstract}
RÉSUMÉ. - L'une des raisons qui expliquent l'intérêt renouvelé pour la philosophie autrichienne, et spécialement pour l'œuvre de Brentano et de ses héritiers, est le fait que les philosophes analytiques ont eu un regain d'intérêt pour les problèmes traditionnels de la métaphysique. Il revient à Brentano, Husserl, et aux philosophes et psychologues qu'ils ont influencés, d'avoir attiré l'attention sur l'épineux problème de l'intentionnalité, qui consiste à rendre compte de la relation entre les actions et les objets ou, plus généralement, de la relation entre les environnements psychologiques des sujets cognitifs et les différents types d'environnements externes (physique, géographique, social) qu'ils habitent. Le présent article s'intéresse à cette version environnementale du problème de l'intentionnalité. Nous nous pencherons non seulement sur les travaux de Husserl et de Scheler, mais aussi sur ceux de Kurt Koffka et de Kurt Lewin en psychologie de la Gestalt. Nous tiendrons compte de l'influente théorie idéaliste subjective des environnements animaux mise de l'avant par J. von Uexküll, que nous comparerons avec une théorie réaliste de l'interaction organisme-environnement, théorie basée sur les écrits des psychologues écologistes J. J. Gibson et Roger Barker. Cette théorie réaliste sera alors exploitée en tant que fondement d'une ontologie des objets sociaux de différents types.
\end{abstract}

\begin{abstract}
One reason for the renewed interest in Austrian philosophy, and especially in the work of Brentano and his followers, turns on the fact that analytic philosophers have become once again interested in the traditional problems of metaphysics. It was Brentano, Husserl, and the philosophers and psychologists whom they influenced, who drew attention to the thorny problem of intentionality, the problem of giving an account of the relation between acts and objects or, more generally, between the psychological environments of cognitive subjects and the different sorts of external (physical, geographical, social) environments which they inhabit. The present essay addresses this environmental version of the problem of intentionality. It draws not only on the work of Husserl and Scheler but also on the Gestalt psychological writings of Kurt Koffka and Kurt Lewin. It considers the influential subjective idealist theory of animal environments put forward by J. von Uexküll and contrasts this with a realist theory of organism-environment interaction based on the work of the ecological psychologists J. J. Gibson and Roger Barker. This realist theory is then exploited as a basis for an ontology of social objects of a range of different sorts.
\end{abstract}

\section{Introduction}

\subsection{Les problèmes de la philosophie}

O n a pu sentir, au cours de l'histoire de la philosophie, deux courants tenaces. D'une part, on trouve la tendance ockhamienne : elle consiste à embras-

1. Traduit par M. H aller, N. Tavaglione et K. M ulligan. 
ser à la fois l'une ou l'autre des ontologies simplifiées tirées d'un petit répertoire, l'atomisme et le monisme par exemple, et un point de vue selon lequel les entités compliquées doivent être, d'une manière ou d'une autre, réduites aux éléments simples acceptés par l'ontologie privilégiée. D'autre part, on trouve le cartésianisme : il consiste à adopter l'une ou l'autre des doctrines épistémologiques fondationnalistes, ou, en d'autres termes, à priser l'épistémé aux dépens de la doxa. Les deux tendances se renforcent mutuellement. Ainsi, le fondationnalisme attire l'attention des philosophes sur les éléments ontologiques simples, car on considère qu'il est plus facile, au sujet de ces derniers, d'atteindre une connaissance indubitable. Les philosophes sont ainsi protégés de tout souci des objets mésoscopiques complexes de notre environnement quotidien et du monde social (ce dernier étant, après tout, un royaume d'opinion pure, indigne des soins deceux qui recherchent la rigueur).

Pas moins que ceux qui travaillaient dans d'autres traditions, les philosophes autrichiens ont été marqués par ces deux tendances. Brentano, tout particulièrement, était un fondationnaliste déclaré et un partisan de l'immanentisme psychologique; et dans sa philosophie tardive, il a embrassé une ontologie selon laquelle tous les objets doivent appartenir à la catégorie unique des choses ou substances. H usserl, de même, défendait l'idée selon laquelle, pour que la connaissance des objets soit simplement possible, ces derniers et les sujets cognitifs doivent être en dernière analyse taillés dans la même étoffe (l'esprit). M ach, comme on le sait, épousait un monisme atomiste (« neutre») des « éléments ». Lui et Brentano étaient fortement influencés par l'héritage sceptique du corpuscularisme, lequel les a conduits à tenir le monde du sens commun (celui des tables et de la bière, des mariages et des batailles, des orchestres et des armées) pour une chimère, un produit de la Falschnehmung ou des rouages inertes de l' « économie de la pensée ». Tous deux ont de sérieuses difficultés à intégrer à leurs vues des structures holistiques ; il est intéressant, sur ce point, de noter que le gestaltisme, inauguré par Christian von Ehrenfels dans son article classique intitulé « Über Gestaltqualitätenä » (1890), était à ses débuts une tentative de forger, à partir des théories de la sensation de $M$ ach et Brentano, une explication efficace de notre perception des mélodies et des autres objets d'expérience complexes.

\subsection{L'environnement psychologique}

N ous nous intéressons ici aux touts sociaux, qui peuvent comprendre des objets sociaux très complexes tels que l'O rdre des chevaliers de la Légion d'honneur, la Guerre de succession espagnole ou le procès 0 .J. Simpson. Les philosophes autrichiens comme $M$ ach, Wittgenstein et G ustav Bergmann, qui s'efforçaient de reconstruire le monde à partir d'éléments simples (par exemple : sense-data, substances, particules, propriétés universelles élémentaires) par le biais d'une agglomération de phénomènes objectifs, de faits, d'ensembles, de classes, de circonstances, de réalités, de possibilités, et ainsi 
de suite, étaient condamnés à l'échec dans leur entreprise d'ancrer théo riquement le type d'intégrité que ces touts manifestent. Ces derniers, en effet, ne sont en rien le résultat d'une quel conque composition logique (ou physique, ou psychologique). M ais ils n'en sont pas moins réels; c'est du moins ce que je veux démontrer. La tradition philosophique inspirée par Brentano, en revanche, incluait un certain nombre de penseurs aux penchants holistes, qui n'étaient pas attachés au réductionnisme logique ou ontologique. Le présent essai raconte l'histoire de la redécouverte graduelle des objets sociaux, ainsi que des objets du sens commun en général (les objets complexes du royaume de l'expérience non-apodictique), par les successeurs de Brentano, d'Ehrenfels et de H usserl. La lutte d'Ehrenfels avec le problème des touts complexes fut reprise par $\mathrm{M}$ einong et par son étudiant $\mathrm{Fritz} \mathrm{H}$ eider, par l'étudiant de Ehrenfels M ax Wertheimer, par Karl Bühler et par Egon Brunswik à Vienne, puis par les grands théoriciens gestaltistes qui se réunissaient autour de $C$ arl Stumpf à Berlin et au nombre desquels on comptait non seulement Wertheimer, mais aussi Wolfgang Köhler, Kurt Koffka et Kurt Lewin. À la suite de leur émigration en A mérique, $\mathrm{H}$ eider, $\mathrm{K}$ offka et Lewin influencèrent à leur tour les écopsychologues J.J. Gibson et Roger Barker². C'est dans les travaux de ces derniers qu'on trouve enfin l'ébauche d'un cadre théorique approprié à l'ontologie des objets sociaux. Sousl'effet del'influence toujours vivace des philosophies atomistes et immanentistes, Stumpf, le premier H usserl, M einong et d'autres contemporains pensaient avoir pour tâche d'expliquer comment l'apparence de la complexité peut surgir d'éléments mentaux simples. Aux yeux de ces philosophes, l'environnement externe du sujet psychologique est une affaire de pure construction. Les gestaltistes tardifs s'intéressèrent plus résolument au monde. Ils le comprenaient dans des termes physiques, mais étaient néanmoins sensibles à la complexité authentique et autonome de la structure de la réalité physique. Q uand ils en vinrent à considérer l'environnement de la perception et du comportement humains, cependant, ils tombèrent dans le piège suivant : ils crurent que l'« environnement comportemental » ou « psychologique » n'existerait qu'en tant que simple aspect d'un tout relationnel englobant même le comportement qui l'a produit.

II est utile, pour comprendre le problème en jeu, de citer les lignes de Koffka dans lesquelles est introduite la distinction gestaltiste entre les environnements « comportemental » (ou mental) et « géographique » (ou physique) :

Une soirée d'hiver qu'une tempête de neige faisait rage, un cavalier parvint à une auberge, heureux d'enfin rejoindre un gîte après avoir chevauché tant

2. Gibson et K offka ont été, pendant un certain temps, les collègues de Smith ; H erbert S. Langfeld, le superviseur de thèse de Gibson, était lui-même un ancien étudiant de Stumpf. Au sujet de ces influences de Gibson, voir Reed, 1989, p. 19 et suivantes, 38, 49 et suivantes. Sur Barker et Lewin, voir Schoggen, 1989, p. 300. 
d'heures sur la plaine balayée par le vent, dont les chemins et les points de repère avaient été effacés par une couverture de neige. Le maître des lieux vint à la porte ; étonné de voir cet étranger, il lui demanda d'où il venait. L'homme fit un signe en direction de la plaine. Sur quoi le maître des lieux, la voix remplie d'effroi et de surprise, répondit: "Vous avez traversé le Lac de Constance, le savez-vous? » À ces mots, le cavalier, mort sur le coup, s'écroula aux pieds de l'aubergiste.

Dans quel environnement le comportement de l'étranger a-t-il donc pris place? Sur le Lac de Constance. A ssurément, et cette localisation particulière intéresse le géographe. $M$ ais elle ne doit pas retenir l'attention d'un psychologue étudiant le comportement. Car celui-ci doit plutôt dire : dans un second sens du mot « environnement », notre cavalier n'a pas du tout traversé le lac, mais il a chevauché sur une banale plaine balayée par la neige. Son comportement consistait à chevaucher-sur-une-plaine et non pas à chevaucher-sur-un-lac. ${ }^{3}$

Ce dont nous faisons l'expérience, selon des gestaltistes tels que K offka, ce n'est pas des objets de la réalité physique (les objets de l'environnement géographique). Bien plutôt, nous faisons précisément l'expérience de G estalten, à savoir d'objets créés qui diffèrent des objets de la réalité physique, entre autres en cela qu'ils résultent de l'application de certaines « lois » spéciales d'organisation gestaltistes.

À l'instar de leurs prédécesseurs brentaniens, par conséquent, les gestaltistes ne concevaient pas l'environnement psychologique dans des termes réalistes. D'emblée, ils n'étaient pas à même de parvenir à une théorie cohérente de la relation entre cet environnement et le monde physique 4 . Des psychologues tels que Lewin, H eider et Brunswik, munis de leurs théories de I' « environnement psychologique », ne purent atteindre une position pleinement réaliste : I'environnement psychologique est, à leurs yeux également, une chose dépendante du moi, présente même dans les rêves 5 .

Chez le Husserl tardif lui-même, on peut découvrir des confusions du même ordre, même si I dées II et $L$ a crise des sciences européennes renferment un éventail de descriptions magistrales des caractéristiques du monde du sens commun $^{6}$. Des méprises similaires se retrouvent aussi chez Scheler, dont la théorie du « milieu » de la vie pratique influença les écrits de H eidegger sur l'« être-au-monde ${ }^{7}$ :

Les « choses » qui importent à notre action - celles par exemple auxquelles nous songeons lorsque nous ramenons telles ou telles conduites humaines (ou telles ou telles dispositions à ces conduites) au « milieu » dans lequel vivent les

3. Koffka, 1935, p. 27 et suivantes.

4. Voir M ulligan, 1995, p. 189 et suivantes.

5. Voir I'essai de Heider, The Description of the Psychological Environment in the Work of $M$ arcel Proust (1989, p. 85-107), particulièrement p. 95 et suivantes.

6. Voir Smith, 1995a pour un survol de l'ontologie husserlienne du monde du sens commun ainsi que de ses problèmes.

7. Cf. M ulligan, 1995. 
hommes en question - il va sans dire que ces « choses » n'ont absolument rien à voir avec ce que Kant appelle « choses en soi », pas davantage avec les objets que considère la science (ceux qu'elle suppose pour «expliquer » les faits naturels). Le soleil-demilieu par exemple, n'est pas le soleil des astronomes ; la viande qui est volée, achetée, etc., n'est pas une somme de cellules et de tissus où se produisent des processus physiques et chimiques. Le soleil-de-milieu n'est pas le même soleil pour l'homme situé au Pôle $\mathrm{N}$ ord, pour celui qui vit dans la zonetempérée, pour l'habitant de l'équateur, et son rayonnement senti n'est pas le même rayonnement pour ces trois hommes (...). Je peux subir, sur le plan objectif, toutes sortes d' «actions-efficaces», par exemple des courants électriques et magnétiques, des rayonnements que je ne perçois pas, etc., et tout cela n'appartient certainement pas à mon «milieu » (...). À mon milieu n'appartient que ce qui est vécu-par-expérience-vécue comme agissant efficacement sur moi. ${ }^{8}$

Le problème soulevé par ce passage est clair. Comme les élèves penchés sur un microscope le savent, la viande que l'on vole ou que l'on achète possède sans aucun doute des cellules et des tissus dotés de propriétés chimicophysiques. Le soleil dont on a l'expérience au Pôle $\mathrm{N}$ ord est certainement le même que celui dont on a l'expérience sous les tropiques. Par conséquent, il ne peut être vrai que les choses appartenant à notre environnement pratique, du sens commun, n'ont « rien à voir » avec les objets admis par la science. $M$ ais Scheler continue : La « chose-de-milieu » appartient à un domaine intermédiaire entre notre contenu-perceptif et les objets qui le constituent, d'une part, et, d'autre part, les objets objectivement pensés ». ${ }^{9}$

\subsection{La biologie constructiviste de Uexküll}

Le discours de Scheler sur les royaumes intermédiaires n'est pas sans rappeler la biologie constructiviste de Jacob von Uexküll, que connaissait Scheler. U exküll, lui aussi, acceptait une ontologie des milieux ou des « environnements » (U mw elten), compris comme des entités relationnelles ou intermédiaires comblant d'une manière ou d'une autre l'abîme séparant l'animal du royaume de la physique. Chaque animal, soutenait U exküll, est le créateur de sa propre « réalité externe », d'un U mwelt quel'organisme construit pour ses propres besoins. Le premier principe de sa théorie de l'U mwelt est : " all animals, from the simplest to the most complex, are fitted into their unique worlds with equal completeness. A simpleworld corresponds to a simple animal, a well-articulated world to a complex one ${ }^{10}$. D'une part, on regarde ces réalités construites comme séparées les unes des autres à la manière des monades leibniziennes (Uexküll lui-même use à leur endroit de

8. Scheler, 1954, p. 158 et suivantes, traduction française p. 157.

9. I bid, p. 159, traduction anglaise p. 140, traduction française p. 157.

10. Uexküll, 1957, p. 10. 
I'expression : « bulles de savon » ${ }^{11}$ ). $\mathrm{D}$ 'autre part, cependant, on reconnaît à ces réalités la capacité d'interagir, même si l'explication de cette interaction est difficileà comprendre. L'œil, nous dit-on, « projette » l'image créée sur sa rétine hors de lui-même, dans l'espace visuel qui environne l'animal. « Si I'œil était privé de cette faculté, la libellule serait incapable d'attraper un moucheron en vol... Le son, l'odeur, le goût et le toucher sont tous transportés hors du corps dans l'espace subjectif de l'animal $\gg 12$.

On raconte que U exküll en est arrivé à cette doctrine quand, contemplant un hêtre dans les bois de $\mathrm{H}$ eidelberg, il se rendit compte de la chose suivante :

Ceci n'est pas un hêtre, mais plutôt mon hêtre - quelque chose que, avec mes sensations, j'ai moi-même construit dans ses moindres détails. Tout ce que je vois, entends, respire ou sens, ce n'est pas des qualités appartenant exclusivement au hêtre, mais bien des caractéristiques de mes organes sensoriels que je projette hors de moi-même. ${ }^{13}$

Reprenons la formulation de la question donnée par U exküll dans sa Biologie théorique ${ }^{14}$ : «Toute réal ité est apparence subjective - ceci doit être également la pierre angulaire de la biologie ».

La saveur kantienne de la pensée de U exküll ressort particulièrement dans une lettre adressée à H ouston Stewart Chamberlain en 1923 : U exküll y parle de l'opposition entre ce qu'il nomme l' « espace intuitif » de l'environnement animal et I' « espace des présentations » (Vorstellungsraum) de la science. Ce dernier, prétend U exküll, ne peut plus revendiquer sa réalité. «Seul est réel l'espace intuitif. ${ }^{15}$ :

Car nous sommes capables de bâtir un représentant conceptuel où soleils et étoiles tournent à des distances incroyables et sur des durées inconcevables. $M$ ais cet espace conceptuel n'est qu'une édulcoration de notre espace concret, que l'on obtient en négligeant certains éléments importants de ce dernier. [...] Je crains, si je rends publique cette perspective, que I'on me traite à la Galilée - que I'on m'enferme dans une maison de fous ou me ridiculise comme un parfait réactionnaire.

11. Et aussi à la manière de tunnels : « If one represents the environment of an animal at a given moment as a circle, then one can add each successive moment as a new environmentcircle. In this way one would obtain a pipe which would correspond in its length to the life of the animal. This pipe will be formed on all sides with characters which one can think of as being built up along and around the life's journey of the animal. This life's journey is thus similar to an environment-tunnel that is closed at both ends. The type of character which can appear in this environment-tunnel is fixed from the start, so that one can designate its breadth and its richness as predestined. But also the temporal length of the tunnel has a predetermined extent, which cannot be exceeded » (Uexküll, 1928, p .70).

12. Jennings, 1909 , p. 333.

13. Schmidt, 1980, p. 10, cité dans H arrington, 1996, p. 41.

14. U exküll, 1928, p. 2.

15. H arrington, 1996, p. 46. «T he world of the physicist counts for the biologist only as a conceptual world [nur als eine gedachte Welt], which corresponds to no reality but which is to be assessed as an aid valuable for computation» (Uexküll, 1928, p. 61). 
Cependant, je me dois d'être, une fois au moins, franc. Personne, peut-être, ne me comprendra. N éanmoins, le fait demeure: «Eppur non si muove. » $\mathrm{Ce}$ n'est pas moi qui tourne autour du soleil : c'est le soleil qui se lève et se couche dans ma voûte céleste. La même chose se passe dans cent mille autres voûtes célestes ${ }^{16}$.

O u, dans une nouvelle veine kantienne: « L'espace doit son existence à l'organisation interne du sujet humain qui habille les qualités sensorielles d'une forme spatiale ${ }^{17}$. 0 u encore :

Au regard du naïf, seul est visible le monde des apparences - lequel, environné par l'espace et le temps, est plein de sons, d'odeurs et de choses colorées. La recherche scientifique s'emploie, en l'attaquant sur deux fronts, à influencer cette vision candide du monde. La théorie physique s'efforce de convaincre le naïf que le monde qu'il voit est saturé d'illusions subjectives, et que le monde réel est bien plus pauvre, lui qui n'est qu'un immense et éternel ballet tourbillonnant d'atomes qui se déploie d'une façon purement causale. La théorie biologique, au contraire, cherche à attirer l'attention du naïf sur le fait qu'il en voit bien trop peu, et que le monde réel est bien plus riche qu'il ne s'en doute: chaque être vivant répand autour de lui son propre monde d'apparences, qui ressemble dans les grandes lignes à celui du naïf, mais qui s'en distingue néanmoins sous tant d'aspects que ce dernier pourrait consacrer sa vie entière à l'étude de ces mondes sans pouvoir pour autant achever l'entreprise. [...] Et quand enfin nous aurons commencé, pour quelques animaux, à montrer quels environnements les entourent comme autant de solides mais invisibles serres, alors nous pourrons bientôt peupler le monde qui nous entoure d'innombrables autres mondes miroitants qui multiplieront par mille les richesses de notre univers. La biologie offre ainsi au naïf l'enrichissement illimité de son monde ; tandis que le physicien le réduit à l'état de mendiant ${ }^{18}$.

\subsection{Le réalisme écologique}

La théorie de la relation animal-environnement développée dans les travaux de Scheler, de Uexküll et des gestaltistes soulève un problème central : elle semble exclure qu'il y ait effectivement un environnement commun aux différentes espèces animales (et rend ainsi inexplicable le fait que la mouche est dévorée par la salamandre). Ce problème est presque résolu par les travaux de Gibson et Barker. O n y trouve une nouvelle étape dans le traitement de la question qui nous intéresse, une étape qui accorde enfin son dû à l'environnement externe. Bien plus que les écrits des gestaltistes les plus radicaux, ceux de Gibson et Barker soulignent lefait quel'expérience psychologique ne doit pas être comprise dans les termes d'une succession de relations bipolaires, intervenant dans quelque royaume spécial, entre des actes et des objets intégrés de manière plus ou moins cohérente. Elle doit plutôt être comprise dans les termes d'un enchevêtrement topologique grâce auquel I'organisme

16. Cité par $\mathrm{H}$ arrington, 1996, p. 46 et suivantes.

17. Uexküll, 1928, p. 4.

18. Uexküll, 1928, p. 62. 
sentant est logé ou situé dans (sert en quelque sorte de frontière intérieure à) un environnement, de telle sorte que ses perceptions et ses actions doivent être théo riquement appréhendées comme des événements prenant place dans ce cadre. Cependant, celui-ci n'est pas conçu dans des termes psychologiques : il tombe nettement dans le domaine de la physique. Bien plus, on admet que nos environnements successifs contiennent des lacs, des chaises, des tables, des salamandres, des plages sablonneuses et des appareils topographiques indépendamment même des croyances relatives particulières que nous entretenons à l'occasion ${ }^{19}$.

Dans la perception comme dans l'action, selon le point de vue de Gibson et Barker, nous sommes entrelacés avec les choses elles-mêmes, et non, par exemple, avec des « sense-data », avec des « représentations » ou avec des « noemata ». Les perceptions, tout comme les actions, sont les accomplissements de créatures douées d'intentionnalité. Partant, la perception n'a rien à faire avec la manipulation psychique des sensations ; elle consiste plutôt en l'acquisition d'une information directe et complexe concernant les objets environnants, information que le sujet obtient grâce au lien que ses activités visuelles, tactiles, gustatives et émotionnelles tissent entre lui et les objets - la chemise en lambeaux, le verre vide, la lance brisée - qui sont pertinents pour sa vie et ses tâches du moment.

Gibson et Barker, comme nous le verrons plus tard, adoptent une conception radicalement externaliste de l'esprit et de l'action. $\mathrm{N}$ otre âme, notre esprit n'est pas cartésien : il n'abrite pas de théâtre intérieur où seraient logés des « contenus », des « représentations » ou des « croyances et désirs ». II échappe par conséquent à la difficulté (qui a infesté le travail de Brentano et de ses successeurs de la première génération) d'expliquer comment cet esprit ou cette âme peut, grâce à l'intentionnalité, saisir des objets qui lui sont extérieurs. Il y a plutôt un organisme percevant et agissant, dont les actions et les perceptions sont toujours déjà inextricablement entremêlées aux parties et aux moments, aux choses et aux surfaces de son environnement externe.

$\mathrm{N}$ i Gibson ni Barker, cependant, ne purent égaler, dans leurs théories de la relation d'enchâssement entre l'organisme et sa niche, la sophistication ontologique que l'on trouve dans les écrits de Brentano, de H usserl ou d'Ingarden ; ils ne disposaient pas non plus des outils de l'ontologie formelle qui semblent pourtant les plus appropriés au traitement systématique des phénomènes holistes qui nous intéressent. Le présent essai constitue ainsi un premier pas en direction d'un remède à ces inadéquations : il donne aux idées des écopsychologues une forme qui permet de mettre à contribution quel-

19. Cette théorie de la perception directe a étéla cible des critiques de Fodor et Pylyshyn, 1981, qui ont beaucoup contribué à assurer que l'œuvre de Gibson demeure mésestimée parmi les philosophes. Ces critiques se rapportent à la question de savoir si l'approche écologique de Gibson peut mener à la découverte de relations nomologiques authentiques dans la sphère psychologique. Elles ne s'adressent pas aux aspects de l'œuvre de Gibson qui sont pertinents à ce qui est discuté ici. 
ques-uns des enseignements que nous avons tirés, avant tout, des récents travaux sur la méréologie et la méréotopologie inspirés de la troisième des Recherches logiques de $\mathrm{H}$ usser ${ }^{20}$.

\section{Une ontologie bicatégorielle}

\subsection{Les continuants et les occurrents}

Comment rendre justice, d'un point de vue ontologique, à la complexité? Comment, plus précisément, des personnes distinctes, comme vous et moi, se réunissent-elles en des touts sociaux divers (comités, équipes, bataillons, réunions, conversations, joutes)? Répondre à cette question, cela requiert que nous distinguions tout d'abord deux catégories d'objets, les continuants et les occurrents, qui servent en quelque sorte de briques de la réalité du sens commun. Les continuants (qui comprennent ce qu'A ristote appelait « substances » ou parfois « choses», « corps » ou « grandeurs étendues ») demeurent identiques à eux-mêmes à travers le temps. Leur existence est continue d'un instant à l'autre et d'un jour au suivant. Voici quelques exemples de continuants : vous et moi, mon rocher préféré, la planète Terre, le lac de Constance, les couches supérieures de l'atmosphère terrestre, le système solaire, Pudding Island, ce que les Anglais appellent «UIster», ce que les Irlandais appellent « UIster » et, dans l'intervalle entre sa formation et son impact sur le sol, une goutte de pluie. Les occurrents (qu'A ristote appelle « accidents » ou parfois « événements », « processus » ou « états ») surviennent ou se déroulent dans le temps ${ }^{21}$. Voici quelques exemples d'occurrents : des sifflements, des rougeurs d'embarras, des courses, mon actuelle migraine, votre connaissance du français, le processus de paix en Irlande du $\mathrm{N}$ ord, I'Intifada, I'hypocrisie actuelle de tel Anglais, le discours d'un Anglais qui vous parle de son sens de l'humour.

Les continuants occupent l'espace et se divisent en parties étendues. Certes, des occurrents tels que le rouge aux joues, les marches funèbres, les feux deforêt peuvent également être étendus dans l'espace ; mais l'étendue et la localisation spatiales des occurrents, dans la réalité mésoscopique du sens commun, sont des parasites del'étendue et de la localisation des continuants qui les supportent.

Un continuant est identique à lui-même du début à la fin de son existence. John enfant est identique à J ohn adulte, malgré les nombreux changements qu'il peut avoir subis au cours des ans. Donc, un continuant n'a pas de parties temporelles : les dix premières années de mon existence font partie de ma vie (à savoir d'un occurrent vaste, complexe et connaissant des phases

20. Voir par exemple Fine, 1995, Johansson, 1989, Simons, 1987, Smith, 1996, Varzi, 1994.

21. Le compte-rendu le plus pertinent de l'ontologie des continuants et des occurrents se trouve dans Ingarden 1964/65. La terminologie est dérivée de Johnson 1921/24. 
multiples) et non pas de moi. Ce ne sont pas les continuants mais bien les occurrents qui peuvent avoir des parties temporelles : il appartient précisément au mode d'existence des occurrents, pour ainsi dire, de se déployer dans le temps. Les parties d'un occurrent comprennent ainsi ses phases successives. Les parties d'un continuant, par opposition, sont ses bras et ses jambes, ses organes et ses cellules. En fait, les deux structures tout/parties (celle des continuants dans l'espace d'une part, et d'autre part celle des occurrents dans le temps) présentent un certain nombre d'analogies profondes ${ }^{22}$. Les continuants comme les occurrents manifestent souvent une structure hiérarchique ou combinatoire à niveaux multiples : un individu est constitué d'atomes, lesquels se combinent pour former des cellules, lesquelles se combinent pour former des organes, lesquels se combinent à leur tour pour former l'individu lui-même ; un match de tennis est constitué de volées, lesquelles se combinent pour former des jeux, lesquels se combinent pour former des sets, lesquels se combinent enfin pour former le match lui-même.

Tandis que les continuants peuvent exister par eux-mêmes, les occurrents ont besoin, pour exister, du support des continuants. Ceux-ci sont les porteurs de ceux-là. Plus précisément, les continuants et les occurrents sont unis par un lien formel de dépendance spécifique. Ce dernier est ainsi défini :

$x$ est spécifiquement dépendant dey $=$ df. (1) $x$ et y ne partagent aucune partie commune et (2) x est nécessairement tel qu'il ne peut exister si y n'existe pas.

M a migraine, par exemple, est spécifiquement dépendante de moi. Le lien formel entre un occurrent et un continuant est uniquement un lien de dépendance spécifique unilatérale. (II est ainsi clair que je ne suis pas spécifiquement dépendant de ma migraine.) On rencontre aussi des cas, cependant, où les objets sont unis par des liens de dépendance spécifique bilatérale. Considérons par exemple la relation entre J ohn le mari et $M$ ary l'épouse, ou entre les pôles nord et sud d'un aimant, ou entre la hauteur, le timbre et le volume d'un son musical ${ }^{23}$. D e même, on rencontre des cas où un complexe de liens de dépendance spécifique unit simultanément un objet à plusieurs autres : des baisers, des poignées de main, des collisions. Certains types particuliers d'occurrents relationnels, que H usserl baptise « moments d'unité » 24 , sont responsables de l'unification, dans un ensemble unitaire unique, d'une pluralité de continuants séparés.

22. Elles sont explorées dans Z emach 1970.

23. Comme N enon (1997, p.102) le souligne, H usserl lui-même n'utiliserait pas ensemble ces différents types d'exemples. Pour lui, des relations comme celle entre mari et femme ou entre roi et sujets sont simplement des « relations corrélatives » gouvernées par des lois analytiques. Toutefois, Z elaniec, 1996 montre que le fait de fixer une limite entre des exemples « analytiques » et « synthétiques » dans cette sphère est loin d'être un problème trivial. Pour notre perspective actuelle, tous les exemples donnés forment un seul continuum. Voir également Zelaniec, 1992.

24. H usserl, 1900/01, traduction anglaise p. 442. 
Les continuants et les occurrents constituent deux ordres de l'être distincts, qui ont joué, ensemble ou séparément, un rôle prédominant dans l'histoire de l'ontologie. D'aucuns, tels A ristote et Ingarden, avaient adopté des ontologies bicatégorielles où les deux ordres avaient leur place. Plus communément, cependant, comme dans le cas de $M$ ach et de Brentano ou de W hitehead et Kotarbinski, on développa des ontologies monocatégorielles qui éliminaient l'une ou l'autre des deux catégories ou en réduisaient l'une dans les termes de l'autre. Ici, en revanche, nous démontrerons qu'il est nécessaire de dével opper une ontologie qui admette, en plus des continuants, des occurrents, de leurs parties et de leurs combinaisons, des objets d'un autre type. Et surtout, avançons-nous, il existe des touts sociaux qui transcendent la frontière entre les deux catégories. Considérons, par exemple, cette institution qu'est la monarchie britannique : elle semble avoir pour parties, à quelque instant qu'on la prenne de sa longue histoire, à la fois des continuants et des occurrents. Les touts de cette nature, qui englobent des objets venus de catégories ontologiques distinctes, nous les appellerons « transcatégoriels ». C es derniers ne peuvent être rangés proprement dans aucune des deux catégo ries, et ont ainsi été négligés par la tradition ontologique.

\subsection{Les occurrents complexes}

Comme nous l'avons vu, les continuants comme les occurrents peuvent se combiner. Équipes, familles, nations sont des exemples de combinaisons de continuants ; réunions, disputes, guerres sont des exemples de combinaisons d'occurrents. N otons en passant que le problème de l'intégrité se pose sous un jour différent pour les combinaisons d'occurrents. En effet, ces derniers ont deux manières de se combiner : premièrement, par composition simultanée - par exemple dans le cas d'un accord musical ou d'un motif coloré - , et deuxièmement, par succession temporelle - comme dans le cas d'une mélodie ou d'une séquence filmée.

Les occurrents peuvent manifester l'unité complexe de divers constituants, cequi ressort clairement de nos expériences perceptives quotidiennes. Comme l'écrit Ehrenfels :

Des exemples tels que la présentation de l'humidité, dans laquelle et le sens de la pression et le sens de la température semblent être également impliqués, ou ces impressions totales que nous désignons de façon imprécise comme les goûts de différents plats mais qui impliquent clairement aussi des sensations de pression, de température et d'odeur, ainsi que d'autres exemples similaires, indiquent que si nous reconnaissons des qualités de forme ( $G$ estaltqualitäten) dans ces sphères, alors, à cause du degré élevé d'unité des complexes présentationnels en question, il faut également accepter la possibilité de qualités de formes qui comprennent des éléments de catégories différentes ${ }^{25}$.

25. Ehrenfels 1890, traduction anglaise p. 97, nous soulignons. 
Certaines combinaisons complexes d'occurrents (par exemple la performance scénique d'un opéra de Wagner) dépendent de combinaisons de continuants. La représentation d'un opéra est une séquence terriblement complexe d'occurrents relationnels, eux aussi complexes, inhérents entre autres aux chanteurs et aux musiciens aussi bien qu'à la scène et à ses soubassements. Comme Ehrenfels l'avait égal ement vu, nombre des réalisations les plus impressionnantes de la créativité humaine ont consistéà découvrir de nouvelles manières de combiner des occurrents simples en occurrents complexes - les « G estaltqualitäten » de Ehrenfels - qui sont alors plus que (et différents de) la somme de leurs parties simples. Les occurrents complexes tels qu'une représentation d'opéra jouissent d'une complexité qui embrasse des constituants venus de domaines matériels extrêmement variés. Une simple promesse manifeste une telle complexité : ellea pour constituants des entités de type à la fois linguistique, psychologique, quasi légal et quasi éthique, aussi bien que des entités plus strictement physiques de différents types (y compris les vibrations de l'air et du tympan, et les événements électriques et chimiques qui y sont associés dans le cerveau).

\subsection{Les continuants complexes}

Les continuants simples peuvent jouir d'une intégrité naturelle : c'est le cas des animaux, des planètes et des gouttes de pluie. Chacun de ces objets bénéficie d'une certaine complétude ou clôture : ni trop petits, ni trop grands (par opposition à leurs parties non détachées (mes bras, vos jambes) et aux amas ou agrégats arbitraires), ils ont des frontières complètes et connectées, et leur position par rapport aux autres objets du monde peut être modifiée.

Les continuants peuvent aussi, cependant, être combinés de façon à former des touts plus ou moins complexes, plus ou moins intégrés, qui sont des pièces authentiques du mobilier du monde. À I'un des extrêmes, nous trouvons des assemblages artificiels tels que la machine à écrire de $M$ einong ou le vaisseau de Thésée ${ }^{26}$. A u milieu, nous trouvons des combinaisons quasi unitaires qui manquent à la fois d'intégrité complète (ils se décomposent facilement) et de clôture complète (leur position n'est pas facilement modifiable). Voici quelques exemples : les plages sablonneuses, le delta des fleuves, les bouquets d'arbres, les chaînes montagneuses. À l'autre extrême, nous trouvons le type d'entités qui nous intéresse ici au premier chef, à savoir les touts sociaux, catégorie richement bigarrée comprenant non seulement les familles et les tribus, les nations et les empires, mais aussi les orchestres et les clubs d'échecs, les bataillons et les équipes de football, en même temps que ces groupements sociaux plus ou moins éphémères qui naissent quand des

26. Les exemples de ce genre, traités par Simons, 1987, seront ici laissés de côté ; leur intégrité peut être admise comme reposant sur de simples facteurs physiques, tels que coller et clouer, et ces problèmes tels qu'ils sont soulevés (se rapportant surtout à la question du principe de leur identité à travers le temps) ne sont pas impliqués ici. 
étrangers sont formellement présentés ou se joignent sur la piste de danse ${ }^{27}$. Ces entités héritent, sinon de toutes, au moins de certaines des marques ontologiques de leurs contreparties simples : elles peuvent au fil du temps subir des changements; elles possèdent une unité qui ressemble à celle d'un être vivant ; elles n'admettent pas de divisions temporelles (les parties de la Pologne sont la Silésie et la Galicie, et non pas la Pologne sous le règne de Sigismond III, la Pologne à l'ère de la partition, et ainsi de suite); elles occupent I'espace; et, de même qu'une chose peut être fendue en parties plus petites, de même, comme l'ont appris les T chèques et les Slovaques, un tout social peut, dans certains cas, être divisé en sous-combinaisons distinctes. Certes, les continuants combinés, à l'image de leurs contreparties simples, sont identiques à eux-mêmes du début à la fin de leur existence; mais cette dernière, I'exemple d'I sraël et de la Pologne le montre bien, peut être intermittente. De plus, le cas de l'A utriche illustre ce point, les touts sociaux peuvent être fondus dans, et séparés, d'autres combinaisons sociales.

Les objets sociaux constituent, au cœur du monde du sens commun, une nouvelle dimension de l'être analogue à celle des individus eux-mêmes. Les institutions vivent leur propre vie, elles perdurent malgré la perte ou l'acquisition de membres; elles possèdent leurs propres qualités, leurs propres états et leurs propres manières de fonctionner en collaboration ou en interaction les unes avec les autres. Et comme les choses appartenant aux niveaux inférieurs, elles sont entièrement dépendantes des circonstances et sont sujettes à des changements dont la structure est plus ou moins régulière et intelligible. La noblesse hongroise existe depuis de nombreux siècles et existera encore pour quelque temps. Les combinaisons de continuants doivent donc être distinguées des ensembles du mathématicien ainsi que des agrégats et des assemblages arbitraires. Ce sont des exemples d'unités survivant au changement.

\subsection{Les objets créés par décret}

Comme le savent les juges, les objets sociaux tels que les jurys, les cours de justice, les contrats et les procès font partie de la réalité. $M$ ais, commel'avait souligné A nton $M$ arty, étudiant de Brentano, ils présentent aussi certaines caractéristiques qu'on associe normalement aux objets du domaine des abstracta ou irrealia ${ }^{28}$. Être réel, sel on $M$ arty, c'est entrer dans des relations causales. L'existence temporelle d'un objet réel implique typiquement des changements continus et multiples, qui reflètent la variété des relations causales où il est engagé. L'existence temporelle d'une combinaison sociale, en revanche, peut être sur de longues périodes exempte de tout changement ; et

27. Voir Gilbert, 1989, 1993, particulièrement p.122 et suivantes de G ilbert, 1993 sur les « sujets pluriels».

28. Pour une discussion des idées de $M$ arty sur les collectifs et les autres variétés d'objets non réels, voir Smith, 1994, p. 96 et suivantes. 
même quand une combinaison sociale subit une modification, celle-ci ne consiste typiquement qu'en sa naissance puis sa disparition sous l'effet de certains changements spécifiques intervenus dans la réalité. $C$ et isolement relatif à l'égard de la sphère concrète et causale-énergétique est caractéristique des objets sociaux dépendants tel s que les revendications, les obligations, les droits, les dettes, les titres de chevalerie, les rapports de propriété et d'autorité, aussi bien que des artefacts culturels tels que les compositions musicales et la littérature.

Chacun de ces derniers est « quelque chose qui, lorsqu'il vient à l'existence, n'est pas produit en tant qu'effet, et qui, lorsqu'il cesse d'exister, ne le fait pas en conséquence de la cessation d'un effet ${ }^{29}$. Les objets sociaux en tant que tels n'ont pas d'histoire faite de changements ; mais ils ne se situent pas pour autant hors de l'histoire: I'existence de la combinaison sociale qu'est la natio hungarica commence avec la création du premier noble magyar et cesse lorsque meurt le dernier noble magyar. L'existence de l'État du M ontana commence avec un certain acte déclaratif accompli à Washington en 1890 et cesse par l'explosion en A mérique de la première bombe cyclotronique lâchée en l'an 2084 par les forces de l'Empire belge en plein déclin. ( $\mathrm{O}$ se rappelle ici la conception leibnizienne des agrégats comme phaenomena bene fundata non réels qui n'appartiennent ni aux substances, ni aux accidents.) Les objets sociaux ont des realia pour parties ; mais ils sont apparemment dans une situation d'isolement (causal) relatif à leur égard, n'étant affectés que par ceux de leurs changements qui sont de nature à entraîner la destruction de la combinaison elle-même.

On pourrait être tenté, avec $M$ arty, d'imposer une structure à deux niveaux au royaume des continuants : au niveau inférieur se trouveraient les choses réelles, sujettes aux changements continuels et aux interactions causales; au niveau supérieur (« survenant ») se trouveraient les combinaisons non-réelles, flottant, pour ainsi dire, au-dessus du niveau réel ${ }^{30}$. $M$ ais cette position pose un problème : ellen'accordeaucune place aux interactions entre les deux niveaux, aux manières dont notre comportement causal-énergétique ordinaire est restreint (comment, nous le préciserons plus tard) par notre participation à des combinaisons sociales et à d'autres types d'institutions. Certes, $M$ arty a raison d'attirer notre attention sur le fait que nombre d'objets sociaux sont les produits d'un décret (c'est là un grain de véritéqu'on trouve dans les doctrines contractualistes dela science politique aussi bien que

29. M arty, 1908, p. 321.

30. Searle avance un point de vue conforme à ces propos, où le niveau des «faits institutionnels » est vu comme étant construit et soutenu par les croyances, les habitudes et les usages de la part des faits impliqués. $\mathrm{N}$ enon, 1997, p.102 semble imputer à $\mathrm{H}$ usserl une telle conception à deux niveaux, mais il tire ici des implications inappropriées de l'usage husserlien du terme « abstrait » comme synonyme de « dépendant » dans la troisième des R echerches logiques, un usage qui n'a rien à faire avec les interprétations standards des abstractas comme entités appartenant à des domaines spéciaux et de niveau supérieur, distincts du domaine des substances concrètes, changeantes et corporelles. 
dans les explications des phénomènes légaux et politiques basées sur la théo rie des actes de langage ${ }^{31}$ ). Ainsi, de nombreux objets sociaux déjà pleinement développés résultent de promulgations légales, de poignées de main, de contrats et d'affirmations. $M$ ais comment réconcilier cet aspect des objets appartenant au domaine social avec leur faculté de restreindre le comportement?

\subsection{La dépendance générique}

De même que les continuants simples peuvent gagner et perdre des parties (comme le chat Tibbles gagne et perd des molécules), de même les combinaisons de continuants peuvent gagner et perdre des membres et subir au cours du temps d'autres types de changements tout en restant identiques à ellesmêmes. Les bourgades, les villes, les universités et les corporations en général présentent la faculté de se maintenir à travers le temps, quand bien même elles enregistrent une certaine rotation de leurs continuants constitutifs : elles peuvent continuer à exister même si certains de leurs participants sont écartés et si d'autres les remplacent. De surcroît, il existe des objets dépendants ayant pour support des continuants ou des touts combinés, mais capables de survivre au remplacement de ces derniers. Les langages, les religions, les systèmes légaux et bien d'autres sortes d'institutions ne dépendent pas, pour exister, d'individus ou de groupes spécifiques; bien plutôt, ils sont en état de dépendance générique à l'égard de l'existence d'individus ou de groupes jouant certains rôles nécessaires.

Pour saisir le sens dans lequel un objet institutionnel dépend d'un continuant, il faut introduire la notion de dépendance générique. On peut la définir approximativement comme suit :

$x$ est génériquement dépendant des objets du type $S=d f$. $X$ est nécessairement tel qu'il ne peut exister sans qu'il existe un objet du type $S$.

Un père, dans ce sens, dépend d'un enfant ; un propriétaire de chien dépend d'un chien ; un roi dépend de ses sujets - mais de nul enfant, de nul chien, de nuls sujets spécifiques. Dans le même sens, un langage, une religion ou un système légal sont génériquement dépendants des individus et des groupes qui, par leurs actions, instancient les règles, les croyances et les coutumes correspondantes. Ce type de dépendance générique, comme nous le verrons, est caractéristique d'objets sociaux de nature très variée.

\section{L'ontologie du monde du sens commun}

\subsection{La théorie des unités physico-comportementales}

Les objets sociaux existent dans cette strate mésoscopique de la réalité que nous nommons le monde du sens commun - le mésocosmos. Ils tombent

31. Reinach, 1913 présente sur cette base une « ontologie des relations sociales » qui fait une place, de façon ingénieuse, non seulement aux décrets socio-institutionnels mais également à la réalité sociale prioritaire à toutes les promulgations et toutes les énonciations de la loi positive. 
ainsi hors du champ des sciences strictement physiques. Le monde du sens commun est un monde où les gens travaillent, conversent, jugent et évaluent ; un monde d'animaux, de tables, de vêtements et de nourriture ; un monde où nous faisons l'expérience du doux et de l'amer, du rouge et du vert, du chaud et du froid. Le monde du sens commun est avant tout le monde des choses que nous utilisons à diverses fins pratiques, des choses qui existent toujours in situ, à savoir dans un environnement peuplé d'autres choses réelles.

En plus des choses, le monde du sens commun contient aussi des trous, les écarts entre les choses, et les media (par exemple l'eau et la fumée) où se meuvent les choses ${ }^{32}$; il contient encore les ombres, les arcs-en-ciel, les marées et les phénomènes similaires. $D$ ans cette vaste collection de choses et de media, on trouve encore des domaines distincts d'organisation qui se recoupent les uns les autres sur un certain nombre de dimensions différentes. Certes, le monde est organisé en choses et en corps séparés ; mais il est aussi organisé en contextes ou en zones sociales et institutionnelles qui se chevauchent et auxquel les les êtres humains participent. $\mathrm{N}$ on pas que nous ayons les individus d'un côté et les contextes de l'autre, séparés par un abîme comblé parl'« intentionnalité »; bien plutôt, pouvons-nous maintenant affirmer, les individus eux-mêmes comme les choses de l'environnement spatial s'insèrent, dans une mesure égale, dans des entités d'un nouveau type inclusif, que l'écopsychologue Barker nomme unités physico-comportementales. Ce sont ces dernières qui servent d'environnements successifs aux individus et aux groupes engagés de jour en jour dans des activités diverses.

Voici quelques exemples des unités physico-comportementales privilé giées par Barker (qui fut l'un des premiers assistants de Lew in à l'Iowa Child Welfare Station) : les cours de W endy le vendredi après-midi, le rendez-vous de Jim avec son professeur, votre déjeuner du mardi, la natation matinale de Frank. De telles unités peuvent être réitérées (peuvent exister en plusieurs exemplaires) :

[Ce] sont des entités phénoménales courantes. Ce sont des unités naturelles en aucun cas imposées par le chercheur. Aux yeux des laïcs, elles sont aussi objectives que les rivières et les forêts - ce sont des parties de l'environnement objectif, lesquelles, à l'instar de la pluie et des plages sablonneuses, font l'objet d'une expérience directe ${ }^{33}$.

Barker insiste sur le fait que les unités physico-comportementales sont des parties de la réalité. Elles sont d'une valeur inestimable pour notre compréhension de l'action et de la cognition humaines : presque tout comportement humain s'y inscrit. Tous les rôles sont joués dans des cadres comportementaux ; toutes les organisations en sont constituées; toutes les biographies sont ordonnées dans leurs termes. Les êtres humains sont de part

32. Voir Fritz H eider, «Thing and M edium», dans H eider, 1959, p. 1-35. Voir également Schoggen, 1989.

33. Barker, 1968, p. 11, je souligne. 
en part déterminés par les cadres comportementaux qu'ils habitent, exactement de même que les animaux non-humains sont de part en part déterminés par les niches écologiques où ils ont évolué. M ême nos voyages d'un lieu à I'autre ou la paresse rêveuse qui entrecoupe nos activités peuvent être reconnus comme des unités physico-comportementales dans les termes de Barker. $M$ ême nos efforts plus ou moins fructueux pour nous lancer dans des entreprises standards ne peuvent être compris en tant que tels que dans les termes de la prédominance indépendante des unités physico-comportementales du type inclusif correspondant : c'est en relation avec ces dernières uniquement que nos efforts sont déterminés en tant qu'efforts et que nous pouvons distinguer nos succès de nos échecs. Les cadres comportementaux dans lesquels nous sommes constamment inscrits, il faut l'admettre, sont dans une certaine mesure poreux, en vertu du fait que nous pouvons parfois déplacer le contexte effectif d'instant en instant selon que notre attention est tantôt distraite par une chose ou un individu, tantôt par une ou un autre. Cela, cependant, ne retire rien à leur réalité, ni à leur caractère saillant, ni à leur pénétration virtuellement universelle dans nos vies d'êtres humains. Ce n'est qu'à de rares moments de désorientation total e que nous semblons affranchis de tout cadre comportemental ; mais cela même implique que c'est en relation avec ces cadres que nous sommes normalement orientés.

\subsection{La préhistoire des unités physico-comportementales}

Si on laisse de côté $\mathrm{H}$ eidegger et les existentialistes français tels que Sartre et $M$ erleau-Ponty (avec leur travail, inspiré de L ew in et d'autres gestaltistes, sur les « projets » etc.), les recherches philosophiques portant sur les unités physico-comportementales sont pratiquement inconnues. La théorie husserlienne du « monde de la vie » est une première approximation informelle de I'ontologie dont nous avons besoin. $M$ ais à $\mathrm{H}$ usserl, également, fait défaut un engagement pleinement réaliste à l'égard de ce qu'il nomme l'« espace environnant »; et la relation de ce dernier avec les choses physiques situées dans l'espace n'est jamais tirée au clair.

La négligence qui, dans la littérature philosophique, frappe les unités physico-comportementales s'explique en premier lieu par la tendance déjà mentionnée à privilégier les ontologies simplifiées (par-dessus tout celles qui soutiennent que les continuants ou les occurrents sont les seules catégories admissibles) et par le penchant à embrasser une forme ou une autre de monisme ontologique. Car les unités physico-comportementales, cela devrait désormais être clair, sont radicalement transcatégorielles. Ce qui a conduit ces philosophes même qui nourrissaient l'ambition de se frotter au royaume du sens commun à terminer leur course avec des théories qui le réduisent à des objets conformes au goût moniste - dans la ligne, par exemple, de la doctrine wittgensteinienne des jeux de langage. En fait, cependant, lelangage lui aussi appartient à ces phénomènes qui ne peuvent recevoir une explication cohérente que dans le cadre d'une théorie ontologique des unités physico- 
comportementales. Expliquer la réalité humaine du sens commun en termes de langage, cela revient à expliquer le tout au moyen d'une partie qui s'est développée relativement tard. Cela revient également à prévenir toute interaction mutuellement bénéfique entre notre compréhension de cette réalité et notre connaissance des êtres humains en tant que créatures biologiques.

La négligence envers les unités physico-comportementales s'explique en second lieu par le fait que ce sont des objets de nature holiste : ils doivent ainsi être abordés avec des instruments de type méréotopologique, et sont ainsi de nouveau étrangers à la vision du monde nourrie par les philosophes contemporains travaillant avec les outils de la logique des prédicats et de la théorie des ensembles. L'ontologie formelle des cadres, des niches ou des unités physico-comportementales, par conséquent, est largement sous-développée, en dépit de la disposition fortement croissante des récents travaux de métaphysique à admettre, au sein de leurs systèmes catégoriels, des objets de type non traditionnels, tels que les artefacts, les mondes actuels et possibles, les moments (les « tropes » ou les propriétés individualisées).

$M$ ais la négligence envers les unités physico-comportementales s'explique en dernier lieu par la domination exercée par les idées fondationnalistes sur l'esprit des philosophes depuis des siècles : les unités physico-comportementales (ma soupe du soir, votre nage du mardi) appartiennent en effet par excellence au royaume de l'opinion pure. En conséquence de quoi on a conclu erronément, ou bien que les objets considérés ne sont pas susceptibles de recevoir un traitement rigoureux, qu'il soit philosophique ou scientifique, ou bien qu'ils n'existent pas (puisque toute « opinion » est fausse).

\subsection{Les propriétés ontologiques des unités physico-comportementales}

Chaque unité physico-comportementale a deux sortes de composantes : premièrement, des êtres humains manifestant certains comportements (prononçant une conférence, s'asseyant, écoutant, mangeant) ; et deuxièmement, les objets non psychologiques requis par ces comportements (chaises, murs, papier, fourchettes, etc.). Chaque unité physico-comportementale possède une frontière qui sépare une structure interne organisée (premier plan) et une structure externe (arrière-plan) - I'horizon de H usserl. Cette frontière ellemême, bien que loin d'être simple, est une partie objective de la nature, qui peut cependant changer en fonction des participants impliqués ou des circonstances du moment. Chaque unité englobe ses composantes, ce qui signifie que la première entoure très précisément (encercle, environne) les secondes : les élèves et le matériel sont dans la classe ; le magasin ouvre ses portes à huit heures et les ferme à dix-huit heures. II est certain quela portion environnante de la réalité $n$ 'est pas physiquement distincte de ses voisines. La portée de cette portion découpée de la réalité est de nature exclusivement psychologique (elle se rattache, en effet, à la psychologie du sens commun) ; mais elle n'en existe pas moins en tant que partie de la réalité physique. 
Les unités ont leur propre comportement, gouverné par des lois qui leur sont également propres et qui diffèrent de celles qui règnent sur le comportement des individus concernés (nouvelle conséquence de la transcatégorialité, qui a beaucoup contribué à rendre les unités physico-comportementales rétives à l'approche scientifique). Pour Barker, la meilleure explication des lois qui gouvernent les unités physico-comportementales est celle qui utilise des termes mécaniques, ou au moins artificiels (lesquels rappelleront notre discussion de la théorie des combinaisons d'objets proposée par Anton M arty) :

Le modèle de la machine paraît plus approprié à la représentation de ce qui se passe [dans le royaume des unités physico-comportementales] que le modèle de l'organisme ou de la personne. Par exemple, cette entité (physico-comportementale) peut être « débranchée » et dispersée à volonté par l'opérateur, le président. II peut ajourner la réunion (pour une pause café) et la convoquer à nouveau. Tandis qu'elle s'est dispersée, certaines de ses parties peuvent être ajustées (un intervenant remplacé). Des propriétés psychologiques de cette nature, les individus n'en ont point ${ }^{34}$.

A insi, I'histoire temporelle d'au moins un grand nombre des unités physico-comportementales qui structurent nos vies n'a pas les mêmes contours que l'histoire temporelle des individus et de leurs expériences respectives. Les unités physico-comportementales ont souvent un début et une fin nets (pensons au début et à la fin d'une course ou d'un accord contractuel). Par opposition, nos douleurs, nos maladies et nos regrets ont la caractéristique de s'amplifier et de s'atténuer. Les unités physico-comportementales et leurs cadres sont aussi parfois marqués par des frontières spatiales plus tranchées et plus souvent rectilignes que les phénomènes naturels tels que les épidémies ou les tempêtes. Cependant, les limites des cadres comportementaux n'ont pas besoin, sous d'autres aspects, d'être tranchées (considérons, par exemple, la question de savoir si l'éternuement du fiancé fait ou non partie de l'unité physico-comportementale qu'est son mariage).

Par ailleurs, les unités physico-comportementales présentent la faculté de se maintenir par elles-mêmes, ce qui les rapproche du royaume biologique. Elles sont typiquement autorégulatrices et peuvent ainsi diriger leurs composantes vers des états caractéristiques et sont à même de limiter les changements qu'ils subissent sous l'effet de perturbations éventuelles ${ }^{35}$. De légères modifications affectant certaines dimensions données de l'unité peuvent être supportées sans que l'existence continue de cette dernière ne souffre aucun préjudice. Le comportement global de l'unité (par exemple une conférence) ne peut être considérablement altéré sans qu'elle soit détruite. La conférence doit comprendre une introduction ; il doit y avoir un discours, une audience et une discussion. La réunion contient des sous-parties : le président, l'inter-

34. Barker, 1978, p. 34 et suivantes.

35. Barker, 1968, p. 154 et suivantes. 
venant, I'interlocuteur et l'audience (de même que dans une phrase, il y a des sous-parties : sujet, verbe, nom, accents toniques, et ainsi de suite).

\subsection{L'ajustement mutuel systématique du comportement et du cadre écologique}

Une unité physico-comportementale est une unité : ses parties sont unifiées, mais pas par une quel conque similarité ou communauté de substance.

Le comportement et les objets physiques qui forment conjointement la totalité d'une unité physico-comportementale donnée sont entremêlés de telle sorte qu'ils présentent une structure qui ne doit rien au hasard : il y a, entre les modes de comportement standards présents dans l'unité et l'organisation de ses composantes physiques, une relation d'ajustement harmonieux (les sièges de l'auditoire font face au conférencier ; ce dernier adresse ses remarques à l'audience ; les limites d'un terrain de football sont aussi les limites du jeu ; le début et la fin d'une leçon de musique marquent les limites du mode de comportement musical). Cet ajustement mutuel du comportement et de l'environnement physique s'étend à la structure intérieure fine du comportement, de telle sorte qu'est impliquée une non-transposabilité radicale des modes établis de comportement d'un environnement à l'autre. Les conditions physiques, historiques ou cérémonielles qui ont cours dans des cadres particuliers sont en outre aussi essentielles à certains types de comportement que les personnes dotées de l'autorité, des motivations et des compétences requises ${ }^{36}$.

Plusieurs forces ou influences aident à produire et à maintenir cet ajustement mutuel; elles constituent l'unité intertemporelle de l'unité physicocomportementale ${ }^{37}$. Les forces allant du cadre au comportement incluent d'abord les contraintes physiques exercées par les haies, par les murs, par les corridors ou par des individus portant matraque; elles incluent ensuite les forces sociales présentes dans l'autorité de l'enseignant, dans les menaces, dans les promesses et dans les mises en garde ; elles incluent encore les effets physiologiques du climat, le besoin d'eau et d'aliments; et elles incluent enfin les effets des traits physiognomoniques perçus dans l'environnement (les vastes étendues peuvent séduire les enfants, une atmosphère affairiste encourage les comportements affairistes). L'ajustement mutuel peut être renforcé par l'apprentissage et par un processus de sélection des individus concernés, qu'il s'agisse d'autosélection (celle des enfants qui continuent à suivre l'école du dimanche s'ils se sentent capables de se conformer aux modes de comportement établis correspondants), ou qu'il s'agisse de tests d'entrée physiques ou mentaux imposés de l'extérieur. Les forces allant dans la direction inverse, à savoir du comportement au cadre, incluent les innombrables manières dont une succession d'actions distinctes et non coordonnées peut

36. Ibid, p. 32.

37. I bid, p. 30 et suivantes. 
produire des conséquences non intentionnelles, sous la forme de nouveaux types d'actions ou de nouveaux types modifiés de cadres ( à l'exemple de ces sentiers formés sur les coteaux par le passage répété de pieds nombreux $)^{38}$. Dans le cas, finalement, des unités physico-comportementales impliquant une grande quantité de participants, il y a des influences qui trouvent leur source dans le pouvoir de contrôle que différents membres exercent à différents degrés sur le fonctionnement de l'unité.

\subsection{L'enchevêtrement hiérarchique}

Beaucoup d'unités physico-comportementales surviennent conjointement, de même qu'un embryon de poule, par exemple, est construit comme une hiérarchie enchevêtrée d'organes, de cellules, de noyaux, de molécules, d'atomes et de particules subatomiques. À la fois les modes de comportement établis manifestés par les individus inscrits dans l'unité et les structures stables des objets physiques associés sont normalement susceptibles d'être encore divisés en sous-unités dotées de leurs propres frontières plus ou moins définies et saillantes:

Une unité appartenant au niveau intermédiaire de la structure d'enchevêtrement est en même temps englobante et englobée, en même temps tout et partie, en même temps entité et environnement. Un organe - par exemple le foie - est un tout par rapport à la structure cellulaire qui le constitue; et c'est une partie en relation avec l'organisme englobant qu'il compose en collaboration avec d'autres organes ; il constitue l'environnement de ses cellules et se trouve lui-même environné par l'organisme ${ }^{39}$.

II peut exister plusieurs unités physico-comportementales de niveau inférieur dans un lieu donné, et elles sont typiquement insérées dans des unités plus vastes. Le tracé d'un triangle sur le tableau noir s'inscrit dans le cours de géométrie, qui s'inscrit dans l'école, qui s'inscrit dans le quartier, et ainsi de suite ${ }^{40}$.

A ux yeux de Gibson, la réalité en général est une hiérarchie complexe de niveaux enchevêtrés : les molécules sont enchâssées dans les cellules, les

38. Le système global des sentiers sur le coteau se pose comme une conséquence non intentionnelle de plusieurs actions entraînées à une échelle locale. Friedrich von H ayek, 1979 démontre à quel degré une série de phénomènes culturels, incluant les lois, le langage, la religion et le marché, doit son origine à une accumulation imprévue d'effets de décisions et d'actions individuelles dans le temps. L'école autrichienne d'économie, de laquelle $\mathrm{H}$ ayek, avec $\mathrm{Carl}$ $M$ enger et $L$ udwig von $M$ ises, est un membre majeur, cherche généralement plutôt à comprendre les phénomènes sociaux de grande échelle dans les termes de leur parties constitutives de petite échelle - ou, en d'autres mots, dans les termes des cadres de comportement dans lesquels les participants individuels sont impliqués. Sur la pluralité des interconnexions entre les idées des philosophes autrichiens et celles des économistes autrichiens, voir Grassl et Smith, dir, 1986. Les idées expliquées ici sur les cadres de comportement et sur I'« accord » del'organisme avec l'environnement sont également reliées à la notion de « créneau de marché » des économistes. Voir Smith, 1986.

39. Barker, 1968, p. 154.

40. I bid, p. 11et suivantes, p. 16, 1978, p. 34. 
cellules sont enchâssées dans les feuilles, les feuilles sont enchâssées dans les arbres, les arbres sont enchâssés dans les forêts, et ainsi de suite ${ }^{41}$. Chaque type d'organisme est accordé, dans sa perception et dans son action, aux objets situés à un niveau spécifique de cette hiérarchie complexe - aux objets qui s'associent pour former ce que Gibson nomme une « niche écologique ». (La théorie que Gibson propose pour expliquer cette relation d'accord - en termes de collecte d'information - ne doit pas retenir ici

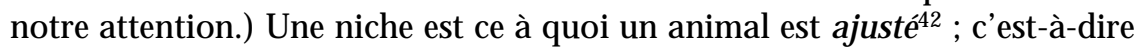
que c'est en rapport avec cette niche que l'animal a développé des habitudes comportementales. Une niche ne comprend pas seulement des choses diverses, mais aussi des formes, des textures, des tendances, des frontières (surfaces ou bords), toutes choses qui sont organisées de manière à être disponibles pour l'animal : elles concernent sa survie. Ces caractéristiques de la niche motivent l'organisme; elles sont de nature à faire intrusion dans sa vie, à le stimuler de multiples manières.

Les perceptions et les actions humaines, pareillement, sont accordées aux formes, aux qualités et aux modes de comportement caractéristiques de nos environnements respectifs (mésoscopiques) ${ }^{43}$. Cette imbrication mutuelle, cependant, s'étend dans notre cas par le biais d'artefacts ainsi que de phénomènes culturels tels que le langage et les institutions connexes, qui comprennent les institutions juridiques, administratives et politiques. A pprendre un langage, c'est aussi accroître l'éventail des objets auxquels nous pouvons spontanément ajuster notre comportement ; c'est ainsi multiplier radicalement les types de niche ou de cadre auxquels nous pouvons spontanément nous ajuster.

$\mathrm{N}$ ous pouvons aussi distinguer, en plus de l'enchevêtrement des unités physico-comportementales, un éventail de cas où les cadres s'influencent mutuellement en l'absence même de tout cadre englobant de niveau supérieur dans lequel ils cohabiteraient. Ainsi, des pays limitrophes (particulièrement des nations en guerre ou engagées dans une querelle de frontières), des entreprises concurrentes ou des gangs de rue s'influencent les uns les autres. Dans certains cas, cette influence mutuelle peut donner lieu à, et être contrô-

41. Gibson, 1986, p. 101.

42. I bid, p. 129.

43. Scheler a également vu le besoin d'accepter le fait que nous soyons dans un certain sens accordé à notre environnement, de manière à ce que nous puissions retirer l'information de ce qui nous environne, même en l'absence de calcul réflexif et conscient : « Ainsi, le « milieu actuel » ne se réduit pas à la série des objets que je perçois quand je marche dans la rue ou quand je suis assis dans ma chambre, mais il comprend aussi tout ce dont je tiens pratiquement compte, en tant que cela est présent ou absent, identique ou différent, par exemple les voitures et les hommes quej'évite (perdu moi-même dans mes pensées ou alors quej'ai les yeux dirigés au loin sur un autre homme). De même le matelot peut « compter » sur une tempête prochaine à partir des variations de son « milieu », sans pouvoir dire que telle modification déterminée (par exemple la formation de nuages, la température, etc.) lui sert de signe pour cette prévision » (Scheler, 1954, p. 159, traduction anglaise p. 140, traduction française p. 158). 
lée (et parfois à distance) par, de nouveaux types d'unités physico-comportementales telles que (le travail) des commissions négociant le tracé des frontières, des associations commerciales, des organisations internationales ( $\mathrm{ONU}, \mathrm{OMC}, \mathrm{BIT}$, etc.), et ainsi de suite. La codétermination réciproque des unités physico-comportementales est également illustrée par les situations où différents laboratoires de par le globe testent les résultats obtenus par les autres ${ }^{44}$. Grâce à la délégation administrative, à la sous-traitance et aux institutions du gouvernement rep résentatif, la portée du fonctionnement effectif des unités physico-comportementales peut, en principe, s'étendre à l'infini, ce qui revient à dire qu'elle peut s'étendre bien au-delà même du champ de ce qui peut être accompli, lors d'interactions directes, par l'action et la perception individuelles. L'expérience de planification centrale menée dans I'Europe communiste démontre, cependant, qu'une telle extension connaît des limites ${ }^{45}$.

\subsection{La transcatégorialité et la dépendance générique des unités physico-comportementales}

Une unité physico-comportementale telle qu'une cérémonie religieuse, un championnat de tennis ou une bataille navale, est un objet complexe composé de positions spatiales et temporelles, de comportements, de choses et de personnes reliés entre eux de façon complexe. Ces composantes peuvent comprendre des éléments artificiels (les bâtiments, les rues, les terrains de football, les livres, les pianos, les bibliothèques, les ponts et la chambre des machines d'un navire) ainsi que des traits naturels (les collines, les lacs, les ondes, les propriétés climatiques, les structures lumineuses et sonores). Ces traits et ces éléments peuvent être combinés de façon extrêmement spécifique, par exemple dans une pièce particulière, dans un bâtiment particulier, à un moment particulier, avec des personnes et des objets particuliers, tous distribués dans une structure tout à fait particulière. En général, cependant, ce qui prévaut dans la sphère des entités physico-comportementales est une forme de dépendance générique : un procès doit être conduit par un juge, mais pas forcément par cejuge-là ; la capitale doit être local isée quelque part, mais pas forcément dans cet endroit-ci (et elle peut être déplacée en temps de guerre).

L'unité physico-comportementale comprend des choses et du comportement, mais elle peut, à travers ceux-ci, comprendre aussi une multiplicité d'autres composantes non physiques. Ainsi, l'unité peut comprendre, par exemple, différents types d'éléments linguistiques, légaux et institutionnels, tous combinés dans l'espace et le temps de façon très spécifique. Les phéno-

44. Dans le second livre de ses I dées directrices, $\mathrm{H}$ usserl utilise des idées très proches de celle-ci pour rendre compte des environnements spéciaux des historiens, des scientifiques et des mathématiciens (voir par exemple Smith, 1995a).

45. Voir Steele, 1992. 
mènes impliqués diffèrent non seulement quant à leur constitution matérielle, mais aussi quant à leur forme ontologique : ils comprennent donc des continuants, des événements, des actions, des états et les relations multiples qui se nouent entre ceux-ci. Comme le dit Barker, qui fait écho à des remarques antérieures de Ehrenfels $s^{46}$ :

L'incommensurabilité conceptuelle des phénomènes, qui constitue un obstacle majeur à l'unification des sciences, ne semble pas troubler les unités naturelles. À l'intérieur des unités plus larges, les choses et événements venant de sciences qui sont conceptuellement de plus en plus étrangères les unes aux autres sont incorporés et soumis à des règles. ${ }^{47}$

En ce qui concerne notre comportement, donc, la diversité la plus radicale de types et de catégories n'empêche pas l'intégration.

\subsection{Les personnes en tant qu'objets sociaux}

La relation entre un participant et un cadre est une relation de codétermination réciproque, et cela à des degrés divers. Chaque participant possède deux positions à l'intérieur de l'unité. II en est d'abord une composante, et contribue donc à former l'unité. II est deuxièmement un individu dont le comportement et dont la nature même d'objet social participant sont partiellement formés par l'unité dont il fait partie (sans que cela affecte son existence continue comme être humain). Schoggen décrit les cadres physico-comportementaux comme consistant en des « constellations hautement structurées et improbables d'objets et d'événements qui contraignent le comportement en accord avec leur propre structuration dynamique ${ }^{48}$. La personne est colorée et formée, et déterminée de part en part par le contexte comportemental du moment. Puisque ce contexte est sujet au changement, il s'ensuit, comme le signale Schoggen, qu'une personne possède plusieurs degrés de force, plusieurs intelligences, plusieurs maturités sociales, plusieurs vitesses, plusieurs degrés de libéralité et de conservatisme, et plusieurs moralités en fonction du contexte particulier de son comportement. Par exemple, une même personne manifestant un degré prononcé de bêtise face à un problème mécanique peut montrer beaucoup de compétence et d'adresse dans des situations sociales ${ }^{49}$. Comme le dit le phénoménologue hongrois Aurel Kolnai, une société humaine n'est pas seulement composée de parties diverses : elle est composée de parties diverses reliées de multiples manières ; ces parties composantes ne peuvent donc que se chevaucher. En d'autres mots, la société humaine consiste en dernière analyse en des individus, mais en ce sens seulement qu'elle se divise en une multitude d'individus en passant par un certain nombre de subdivisions sociales, de telle sorte qu'elle comprend le même individu encore et

46. Ehrenfels, 1890, traduction anglaise p. 110.

47. Barker, 1968, p. 155.

48. Schoggen, 1989, p. 4.

49. Schoggen, 1979, p. 7. 
toujours en accord avec ses diverses affiliations sociales, certaines étant factuelles, naturelles et statistiques, d'autres étant en grande partie ou entièrement le résultat de choix volontaires ${ }^{50}$. Une société est ainsi composée des membres d'une communauté, de mariages et de familles, de classes sociales, de cantons, del'État, del'Église, et ainsi de suite. À chacune de ces affiliations correspondent, dans la vie de chacun d'entre nous, différentes zones de saillance et de motivation, différents traits des unités physico-comportementales dans lesquelles nous sommes impliqués.

De même que les parties (non détachées) des continuants non collectifs (tels que votre bras et ma jambe) possèdent des traits particuliers, de même les participants aux continuants collectifs, tels que les acteurs, les amiraux, les astronomes et les artistes, possèdent des traits particuliers analogues. Un être humain ne fonctionne pas comme une partie d'un objet social, dans le sens méréologique le plus simple; mais plutôt, en vertu des différents rôles qu'il occupe, comme un membre ou un élément. N ous pouvons fournir une analyse provisoire de ces traits en faisant appel à l'analyse que donne Brentano, dans sa Théorie des catégories, de ce qu'il appelle les « extensions modales » des substances ordinaires. Celles-ci, selon Brentano, peuvent être transformées de façons différentes en de nouveaux types d'objets (par exemple par le biais de leur engagement dans des occurrents de différents types). Si J ean est en train de courir, alors J ean le coureur est un continuant modifié (de courte durée) de cette espèce. Si J ean est marié, alors J ean le mari est, de la même façon, un continuant modifié (d'une durée normalement un peu plus longue) d'une espèce (institutionnelle) différente. Kit Fine ${ }^{51}$ a nomméles objets de cette espèce « qua objects » (en référence à la terminologie des ontologistes antérieurs: " Jean qua coureur », «Bill qua président», « Socrate quatenus philosophe», etc.).

Dans notre perspective actuelle, J ean qua coureur est, pendant le temps que dure son existence, la somme méréologique de Jean et de la phase actuelle del'acte de courir qu'il est maintenant en train d'accomplir. J ean qua mari est la somme méréologique de Jean et d'un occurrent institutionnel ; mais celui-ci n'est pas uniquement relié à J ean : il est une partie essentielle d'un tout institutionnel plus vaste qui comprend des éléments légaux, religieux, sociaux, fiscaux et biologiques qui sont reliés à une suite d'unités physico-comportementales. Le mari J ean, pouvons-nous dire, est la somme de J ean et de sa portion du lien institutionnel total qui, pour la période en question, I'attache à son épouse. Si leur mariage est annulé, J ean le mari et M ary l'épouse cesseront tous les deux, instantanément, d'exister.

\subsection{De la psychologie écologique au droit international}

$N$ otre théorie des objets sociaux peut maintenant recevoir la formulation suivante. II y a des unités physico-comportementales, des structures durables

50. Kolnai, 1981, p. 319.

51. Fine, 1982. 
de comportement et d'environnement physique, dans lesquelles nous sommes tous impliqués dans nos activités quotidiennes. De telles unités font autant partie du mobilier de la réalité que les continuants et les occurrents ordinaires (tels que vous et moi). II s'agit ici, si l'on veut, d'une donnée préanalytique de la théorie. Ces unités possèdent des parties, par exemple, le Président J ean (lors de la séance d'une commission) et le golfeur J ean (sur le terrain de golf). Elles ont des conséquences : les contrats signés, les ordres donnés, les jugements prononcés, les médailles décernées.

Q uelques unités de ce type forment des chaînes élargies : elles sont des instanciations répétées de structures comportementales identiques ou proches en progression historique, qui sont telles que leurs parties et conséquences jouissent elles aussi d'un statut qui semble transcender toute instanciation particulière. Le Président Bill est Président même quand il dort. Les frontières du Luxembourg restent les frontières du Luxembourg même si elles ne sont plus physiquement marquées ni contrôlées par la police.

Ces unités font partie de la réalité. Elles ont pour parties des choses physiques et des comportements. Le cadre physique d'une telle unité (par exemple, le siège de la Bourse) existe même quand le comportement pertinent n'a plus lieu. M ais l'unité elle-même (le marché boursier durant un jour ouvrable) exige un comportement pertinent pour exister. Dans la sphère du comportement animal, de façon analogue, le cadre d'une unité physico-comportementale (la niche écologique) continue à exister même quand, par exemple lorsque les oies sont parties, aucun comportement du type pertinent n'a lieu. M ais l'unité elle-même (les sites de nidification en saison de nidification) exige un comportement du type pertinent pour exister.

Pour voir comment cette théorie pourrait fonctionner, considérons le cas des objets par fiat dans le domaine spatial, c'est-à-dire les objets qui surgissent grâce au fait quel'on a tracé des frontières spatiales qui, par exemple, séparent deux parcelles de territoir $e^{52}$. Les frontières nationales ainsi que les frontières d'un comté fournissent des exemples de limites créées par fiat dans ce sens, au moins dans ces cas où, comme le Colorado, le Wyoming ou I'Utah, elles ne correspondent à aucune discontinuité qualitative de la réalité sous-jacente. La Floride, les États-Unis, I'hémisphère $\mathrm{N}$ ord et ainsi de suite sont des objets par fiat de nature géographique.

$O r$, de tels objets par fiat sont déterminés de fond en comble par les unités physico-comportementales auxquelles ils sont associés. Une parcelle immobilière est ce qu'elle est et où elle est (et distincte en nature de toute parcelle sous-jacente de territoire physique) en vertu d'actions qui sont accomplies dans les services cadastraux et lors de recherches géo désiques. Une nation est ce qu'elle est et où elle est en vertu d'actions qui sont accomplies dans les ministères, dans les chancelleries et dans les avant-postes militaires. Une œuvre d'art est ce qu'elle est et où elle est en vertu d'actions qui son

52. Voir Smith, 1995, Smith et Varzi, 1997. 
accomplies dans les bureaux des historiens de l'art, des galeristes, des conservateurs et dans les ateliers de restauration. Chacun de ces processus d'authentification peut évidemment tomber en panne. Ainsi, par exemple, les activités d'un escroc dans le domaine immobilier peuvent créer des unités physico-comportementales fausses ou inauthentiques que beaucoup n'arrivent pas à distinguer des unités vraies. J ackso ${ }^{53}$ montre comment le système étatique de droit international (fondé sur le principe de reconnaissance mutuelle) peut même conférer le statut d'État à des « quasi-États » dont le gouvernement putatif n'assure pas le contrôle intérieur du territoire en question. De soi-disant galeristes ont accordé une crédibilité fallacieuse à plusieurs genres de quasi-art. Encore une fois, cependant, la possibilité même de telles pannes présuppose le fonctionnement correct du système des unités physico-comportementales dans le cas normal.

\section{En défense du réalisme}

\subsection{Gibson est-il réaliste?}

II y a une similarité apparente entre la perspective écologique de Gibson et les théories phénoménologiques de la L ebensw elt, del'U mw elt, du « milieu » ou del' « espace corporel », avancées non seulement par $\mathrm{H}$ usserl et d'autres phénoménologues, mais aussi par des biologistes constructivistes tels que von U exküll. Cette similarité pourrait nous amener à mettre en doute la thèse, que nous avons acceptée jusqu'ici, selon laquelle G ibson doit être classé parmi les réalistes. $K$ atz ${ }^{54}$ nous prie de considérer les énoncés caractéristiques suivants, tirés de l'E cological A pproach to Visual Perception de Gibson :

L'animal et l'environnement constituent un couple inséparable. Chaque terme implique l'autre. Aucun animal ne peut exister sans environnement. Également, mais de façon moins évidente, un environnement implique un animal (ou au moins un organisme) dont il soit l'environnement ${ }^{55}$.

Les affordances de l'environnement sont ce qu'il offre à l'animal, ce qu'il fournit, pour le bien ou pour le mal. Par [affordance], je veux dire quelque chose qui se réfère et à l'environnement et à l'animal d'une façon qui n'est impliquée par aucun terme existant. Elle implique la complémentarité de I'animal et de l'environnement ${ }^{56}$.

[U]ne affordance n'est ni une propriété objective ni une propriété subjective ; ou elle est les deux, si vous préférez. Une affordance est orthogonale à la dichotomie subjectif/objectif. Elle est à la fois physique et psychique, et ni l'un ni l'autre ${ }^{57}$.

53. Jackson, 1990.

54. Katz, 1987.

55. Gibson, 1979, p. 8.

56. Ibid, p. 127.

57. Ibid, p. 129. 
Ces passages exigent, selon $\mathrm{K}$ atz, une lecture relativiste selon laquelle, comme pour von U exküll, les différentes espèces vivent dans des mondes différents :

Les animaux terrestres perçoivent-ils l'eau correctement et les espèces aquatiques incorrectement, ou vice et versa? Gibson, en tant que relativiste, nous dit que non. Chaque espèce vit dans un monde différent et, de façon complémentaire, chaque animal perçoit de manière différente. L'eau est une substance dans un monde, et un médium dans l'autre. Elle n'est pas absolument une substance, ni absolument un médium. "L'animal et son environnement sont des termes réciproques ». $0 \mathrm{n}$ ne pourrait jamais dire ce qu'est l'eau sans dire pour qui c'est de l'eau, et inversement ${ }^{58}$.

II y a deux motivations principales pour les théories représentationnistes et relativistes de la cognition : (1) le problème de l'erreur, et (2) le problème de l'incompatibilité globale apparente entre différents systèmes de représentation.

L'erreur perceptuelle indique, selon le point de vue standard, que la perception ne peut pas être uniquement le produit des inputs. Elle nous indique que, dans certains cas au moins (par exemple celui de l'hallucination), les objets perceptuels sont créés ou constitués par le sujet percevant. Le relativiste maintient que le monde donné dans la perception est toujours un monde construit ou constitué dans ce sens. Le relativiste est donc capable de résoudre le problème del'erreur sans abandonner le but d'une théorie unifiée de la perception, mais seulement au prix, lourd, de couper les racines de sa théorie dans le seul monde réel qui, du point de vue du sens commun, sert d'environnement à la perception. La solution du réaliste au problème de l'erreur nie que ce qui est phénoménologiquement vécu, comme le phénomène unitaire de «perception », soit en réalité un phénomène unitaire du point de vue ontologique. La tâche de fournir une théorie de la perception (de la perception réussie, véridique, liée au monde) est donc à distinguer de cette autre tâche, tout à fait différente, qui consiste à fournir une analyse de l'erreur perceptuelle (des différents types de déviation du cas standard et véridique).

La seconde motivation du relativisme pourrait être formulée ainsi : l'espace perceptuel du sens commun possède, semble-t-il, une structure euclidienne ou une structure assez proche; I'espace du physicien possède une autre structure, très différente; et il se peut que les espaces perceptuels des souris et des araignées possèdent encore d'autres structures. II ne peut être le cas que toutes ces structures soient vraies de l'espace tel qu'il est en soimême. Donc, continuel'argument, notre espace perceptuel, ainsi que ceux de la souris et de l'araignée, ne sont que des « représentations ». C'est une conclusion constructiviste, relativiste, projectiviste et kantienne de ce type que Katz attribue à Gibson.

58. Katz, 1987, p. 120. 
$M$ ais, si nous restons sur l'exemple préféré de Katz, l'espace (comme nous pouvons le supposer ici) est un continuum. Comme chaque continuum, il peut être partagé de plusieurs façons mutuellement incompatibles (comme un fromage peut être coupé pour produire des morceaux triangulaires, rectangulaires ou circulaires, mais pas simultanément). Tous les membres d'une familled'« espaces perceptuels » mutuellement incompatibles peuvent s'avérer compatibles, après tout, si on les interprète comme des expressions de partitions distinctes, par exemple des partitions, à des niveaux différents de granularité, d'une seule et même réalité. Le monde (comme un fromage) peut être coupé en morceaux de plus d'une façon. L'avantage de la méréologie comme outil de l'ontologie est qu'elle nous montre comment il en est ainsi.

Chaque langage, chaque théorie, chaque système de comportement animal génère sa propre partition globale de la réalité, dira-t-on de ce point de vue. Les différents systèmes de comportement animal génèrent des partitions correspondantes de la réalité en niches écologiques. La perception et le comportement humains génèrent ensemble cette partition mésoscopique de la réalité que nous appelons le monde du sens commun.

Une science des environnements humains présentera un visage bien différent de celui d'une science standard. Ceci a conduit quelques philosophes et chercheurs en sciences cognitives à supposer que les environnements, les cadres et les unités physico-comportementales ne sont que des «phénomènes », que ce sont des constructions subjectives qui doivent être abordées à l'intérieur d'une psychologie représentationnaliste ou d'une psychologie prenant au sérieux le « solipsisme méthodologique ». Le défi, comme Gibson l'a bien vu, est de démontrer comment une science des cadres environnementaux peut être « consistante avec la physique, la mécanique, l'optique, l'acoustique et la chimie», puisqu'elle n'est qu'une science qui porte sur « des faits d'ordre supérieur qui n'ont jamais été rendus explicites par ces sciences et sont passés inaperçus ${ }^{59}$. Pour relever ce défi, il faut développer une théorie réaliste des unités physico-comportementales et des autres types d'objets par fiat qui appartiennent à la cognition humaine quotidienne, sans rejeter la physique quantitative standard. Gibson emploie le terme « écologie », précisément pour désigner la discipline qui devrait comprendre ces faits de niveau intermédiaire; il présente l'écologie comme « un mélange de la physique, de la géologie, de la biologie, de l'archéologie et de l'anthropologie, mais avec un effort d'unification », discipline provoquée par la question suivante: qu'est-ce qui peut stimuler l'organisme? ${ }^{60}$

Comment faire tout cela, c'est maintenant, jel'espère, clair. Les unités physico-comportementales (et leurs contreparties animales) et les objets sociaux avec lesquels elles se combinent sont des parties du continuum spatio-temporel de la réalité, de la même réalité que décrit la physique. Elles

59. Gibson, 1979, p. 17.

60. Gibson, 1996, p. 21. 
sont, dans la perspective de la science physique, des parties délabrées de cette réal ité, des parties quel'on ne pourra jamais comprendre commeles produits $d^{\prime}$ une combinaison quelconque de plots physiques. $M$ ais elles sont néanmoins des parties de la réalité spatio-temporelle.

\section{Bibliographie}

Barker, Roger G., Ecological Psychology. Concepts and Methods for Studying the Environment of H uman Behaviour, Stanford, Stanford University Press, 1968

Barker, Roger G. et al., H abitats, Environments, and $\mathrm{H}$ uman Behaviour. Studies in E cological Psychology and Eco-Behavioural Science from the Midwest Psychological Field Station, 1947-1972, San Francisco, J ossey-Bass Publishers, 1978

Brentano, Franz, Kategorienlehre, Kastil, A., dir., H amburg, M einer, 1933

(traduction anglaise: Chisholm, R. M, Guterman, N., The Theory of

Categories, The $\mathrm{H}$ ague/Boston/London, N ijhoff, 1981)

Brentano, F., Philosophische Untersuchungen zu Raum, Zeit und Kontinuum, Körner, S., Chisholm, R. M ., dir., H amburg, M einer, 1976 (traduction anglaise: Smith, Barry, Philosophical Investigations on Space, Time and the Continuum, London, Croom H elm, 1988)

Brunswik, Egon, Wahrnehmung und $\mathrm{G}$ egenstandswelt. G rundlegung einer Psychologie vom G egenstand her, Leipzig/Vienne, Deuticke, 1934

Fine, Kit, "Acts, Events, and Things», dans Leinfellner, W., et al., dir., Language and O ntology, Vienne, Hölder-Pichler-Tempsky, 1982, p. $97-105$

Fine, Kit, «Part-W hole», dans Smith et Smith, dir., 1995, p. 463-486

Fodor, Jerry, Pylyshyn, Z enon W., "H ow Direct is Visual Perception? Some Reflections on Gibson's 'Ecological Approach' », Cognition, 9, 1981, p. 139-196

Gibson, J. J., The Senses Considered as Perceptual Systems, London, George Allen and Unwin, 1966

Gibson, J. J., The Ecological Approach to Visual Perception, Boston, H oughton-M ifflin, 1979 (réédition : H illsdale, N J, Law rence Erlbaum, 1986)

Gilbert, M argaret, O n Social Facts, N ew York, Routledge, Chapman and $\mathrm{H}$ all, 1989

Gilbert, M argaret, «Group M embership and Political Obligation », The M onist, 76, 1993, p. 119-131

Grassl, Wolfgang, Smith, Barry, dir., Austrian Economics: H istorical and Philosophical Background, New York, New York University Press, London/Sydney, Croom H elm, 1986

$\mathrm{H}$ arrington, Anne, Reenchanted Science. Holism in German Culture from Wilhelm II to Hitler, Princeton, N J, Princeton University Press, 1996 
Hayek, F. A., Law, legislation and liberty : a new statement of the liberal principles of justice and political economy, Chicago, University of Chicago Press, vol. III, 1979.

Heider, Fritz, On Perception and Event Structure, and the Psychological Environment, Selected Papers (Psychological Issues, Vol. 1, N o. 3), $\mathrm{N}$ ew York, International Universities Press, 1959

H usserl, Edmund, Logische Untersuchungen, 2 vol., Halle a. d. S., M ax N iemeyer, 1900/01; seconde édition: Logical Investigations, 2 vol., 1913/21 (traduction anglaise de la seconde édition: Findlay, J. N., London, R outledge and Kegan Paul)

Husserl, Edmund, Ideen zu einer reinen Phänomenologie und phänomenologischen Philosophie. Zweites Buch : Phänomenologische Untersuchungen zur Konstitution, Biemel, M ., dir., 1952 (traduction anglaise: Rojcewicz, R., Schuwer, A., Ideas Pertaining to a Pure Phenomenology and to a Phenomenological Philosophy. Second Book : Studies in the Phenomenology of Constitution, Dordrecht, Kluwer, 1989)

Husserl, Edmund, Die Krisis der europäischen Wissenschaften und die transzendentale Phänomenologie. Eine Einleitung in die phänomenologische Philosophie, Biemel, W., dir., 1954 (traduction anglaise: Carr, David, The Crisis of European Sciences and Transcendental Phenomenology: An Introduction to Phenomenological Philosophy, Evanston, Northwestern University Press, 1970)

Ingarden, R., Der Streit um die Existenz der Welt, 2 vol., Tübingen, N iemeyer, 1964/65 (traduction anglaise partielle du premier volume: Springfield, Thomas, 1964)

Jackson, Robert H. Q uasi-States: Sovereignty, International Relations and the Third World, Cambridge, Cambridge University Press, 1990

J ennings, $\mathrm{H}$ erbert Spencer, "The Work of J. von U exküll on the Physiology of M ovements and Behaviour», The Journal of Comparative N eurology and Physiology, 19, 1909, p. 313-36

Johansson, Ingvar, O ntological Investigations. An Inquiry into the Categories of $\mathrm{N}$ ature, $\mathrm{M}$ an and Society, London, R outledge, 1989

Johnson, W. E., Logic, 3 vol., Cambridge, Cambridge University Press, 1921/ 24

Katz, Stuart « Is Gibson a R elativist? », dans Costall, A., Still, A., Cognitive Psychology in Q uestion, Brighton, H arvester, 1987, p. 115-127

Koffka, Kurt, Principles of Gestalt Psychology, London, Routledge and Kegan Paul, 1935

Kolnai, A urel, «Identity and Division as a Fundamental Theme of Politics », dans Smith, B., dir., Structure and G estalt. Philosophy and Literature in Austria-Hungary and $\mathrm{Her}$ Successor States, Amsterdam, John Benjamins, 1981, p. 317-346 
$M$ arty, Anton, Untersuchung zur G rundlegung der allgemeinen G rammatik und Sprachphilosophie, volume 1, Halle a. S., M ax N iemeyer, 1908 (réédition : H ildesheim, O Ims, 1976)

M ichaels, Claire F., Carello, Claudia, Direct Perception, Englewood Cliffs, Prentice-H all, 1981

M ulligan, Kevin, «Perception », dans Smith et Smith, dir., 1995, p. 168-238 Reed, Edward S., James J. G ibson and the Psychology of Perception, N ew $\mathrm{H}$ aven/L ondon, Yale University Press, 1989

Reinach, Adolf "Die apriorischen Grundlagen des bürgerlich Rechtes», J ahrbuch für Philosophie und phänomenologische Forschung, 1, 1913 (traduction anglaise: Crosby, J., «The A Priori Foundations of Civil Law », A letheia, 3, 1983, p. 1-142)

Scheler, $M$ ax D er Formalismus in der Ethik und die materiale Wertethik, $4^{\text {th }}$ edition, Bern, Francke, 1954 (traduction anglaise : Frings, M. S., Funk, Roger L., Formalism in Ethics and Non-Formal Ethics of Value, Evanston, N orthwestern University Press, 1973)

Schmidt, Jutta, D ie U mw eltlehre Jakob von U exkülls in ihrer Bedeutung für die Entwicklung der Vergleichenden Verhaltensforchung, Inaugural Dissertation, University of M arburg, 1980

Schoggen, P., Behavior Settings. A Revision and Extension of Roger G. Barker's Ecological Psychology, Stanford, Stanford University Press, 1989

Searle, John R., The Construction of Social Reality, N ew York, The Free Press, 1995

Simons, Peter M., Parts. An Essay in O ntology, Oxford, Clarendon Press, 1987

Smith, Barry, «Austrian Economics and Austrian Philosophy », dans Grassl et Smith, dir., 1986, p. 1-36

Smith, Barry, Austrian Philosophy. The L egacy of Franz Brentano, Chicago/ LaSalle, O pen Court, 1994

Smith, Barry, "On Drawing Lines on a M ap », dans Frank, Andrew U., Kuhn, Werner, dir., Spatial I nformation Theory. A Theoretical Basis for GIS (Lecture N otes in Computer Science 988), Berlin/H eidelberg/N ew York, Springer, 1995, p. 475-484

Smith, Barry, "Common Sense», dans Smith et Smith, dir., 1995a, p. 394436

Smith, Barry, «M ereotopology : A Theory of Parts and Boundaries », D ata and Knowledge Engineering, 20, 1996, p. 287-303

Smith, Barry et Smith, David Woodruff, dir., The Cambridge Companion to H usserl, Cambridge, Cambridge University Press, 1995

Smith, Barry etVarzi, Achille C., "Fiat and Bona Fide Boundaries: Towards on O ntology of Spatially Extended O bjects », CO SIT ' 97 : Conference on Spatial Information Theory (Springer Lecture Notes), Berlin/ H eidelberg/N ew York, Springer Verlag, 1997 
Steele, David, Ramsay From M arx to M ises. Post-Capitalist Society and the Challenge of E conomic Calculation, La Salle, O pen Court, 1992

Varzi, A chille C., « On the Boundary between $M$ ereology and Topology », in Casati, R., Smith, B. et White, G., dir., Philosophy and the Cognitive Sciences, Vienne, H ölder-Pichler-Tempsky, 1994, p. 423-442

Von Ehrenfels, Christian, « Über 'Gestaltqualitäten' », Vierteljahrsschrift für wissenschaftliche Philosophie, 14, p. 242-292, 1890 (traduction anglaise: "On 'GestaltQualities' », dans Barry Smith (dir.), Foundations of G estalt Theory, M unich/Vienne, Philosophia, 1988, p. 82-123)

Von Uexküll, Jacob, Theoretische Biologie, Berlin, J. Springer, 1928

Zelaniec, W., The Recalcitrant Synthetic A Priori, Lublin, Artom, 1996.

Zelaniec, W., «Fathers, Kings, and Promises: H usserl and Reinach on the A Priori », H usserl Studies, 9,1992, p. 147-177.

Zemach, E. M., «Four Ontologies», Journal of Philosophy, 76, 1970, p. $213-247$ 